\title{
Mejora de la resistencia al desgaste de aleaciones de aluminio mediante recubrimientos obtenidos por proyección térmica HVOF
}

\author{
J.A. Picas", A. Forn", R. Rilla" y E. Martín" \\ Resumen El objetivo de este trabajo es investigar la posibilidad de mejorar la resistencia al desgaste \\ de las aleaciones de aluminio mediante la utilización de recubrimientos de $\mathrm{CCr}-\mathrm{NiCr}$ y \\ WC-Co-Cr depositados por proyección térmica, HVOF (High velocity oxy-fuel). La \\ microestructura y adherencia de los recubrimientos se ha analizado por microscopía óptica \\ y electrónica (SEM). Las características mecánicas de los recubrimientos se han \\ determinado mediante ensayos de ultra-microdureza y el análisis tribológico (coeficiente de \\ fricción y resistencia al desgaste) se ha realizado empleando un tribómetro Pin on disc (con \\ y sin lubricación).
}

Palabras clave Proyección térmica HVOF. CrC-NiCr. WC-Co-Cr. Tribología.

HVOF thermal spraying on aluminium alloys to improve wear behaviour

\begin{abstract}
This project is concerned with the investigation of the capability of HVOF thermal spraying to improve wear properties of aluminium alloys. $\mathrm{CCr}-\mathrm{NiCr}$ and $\mathrm{WC}-\mathrm{Co}-\mathrm{Cr}$ were tested as coating materials. The adherence and coating microstructure were characterised by optical and SEM microscopy. The ultra-microindentation technique was applied to measure the mechanical properties of the coating. Experiments using a tribometer (pin on disc configuration) under lubricated and dry conditions have been performed in order to evaluate the friction and wear properties of the different coatings.
\end{abstract}

Keywords

HVOF thermal spraying. CrC-NiCr. WC-Co-Cr. Tribology.

\section{INTRODUCCIÓN}

Los recubrimientos obtenidos por proyección térmica HVOF se utilizan en la industria aeronáutica y aerospacial y cada vez son más numerosas sus aplicaciones en la industria del automóvil ${ }^{[1}$ y 2]. El empleo de materiales cada vez más económicos, equipos de proyección más modernos y técnicas de aplicación robotizadas, permite el uso de estos recubrimientos en componentes de automoción, a un coste competitivo. Estos factores, combinados con la necesidad de mejorar la eficiencia y prestaciones del motor, reduciendo el peso de vehículo y controlando las emisiones de gases, están propiciando la utilización de estos recubrimientos sobre materiales ligeros con la finalidad de mejorar sus características superficiales.

Cabe remarcar que las características del proceso de proyección HVOF (baja temperatura y velo- cidad supersónica de las partículas), evita que el substrato a recubrir alcance elevadas temperaturas, permitiendo la deposición de recubrimientos en materiales con temperaturas de fusión relativamente bajas. Este hecho, posibilita el empleo de los recubrimientos HVOF sobre aleaciones de aluminio con la finalidad de mejorar sus características superficiales y su resistencia al desgaste.

En general los recubrimientos basados en el sistema WC-CoCr presentan una elevada dureza y buena resistencia al desgaste, si bien, estos recubrimientos experimentan procesos de decarburización a temperaturas superiores a $450{ }^{\circ} \mathrm{C}$, comportando la degradación de sus propiedades (descomposición del WC en $W_{2} C, W_{3} C$ y W metálico $)^{[3]}$. Por otra parte, los recubrimientos basados en el sistema $\mathrm{CrC}-\mathrm{NiCr}$, si bien presentan durezas algo inferiores, muestran una mayor estabilidad de los $\mathrm{CrC}$ con la temperatura (superior a $800{ }^{\circ} \mathrm{C}$ ) y una

(*) Centro de Diseño de Aleaciones Ligeras y Tratamientos de Superficie (CDAL). Universitat Politècnica de Catalunya (UPC). 08800 Vilanova i la Geltrú.

(**) RALSA (Rilla, Alvarez y López, S.A.). 33930 Langreo. 
mejor resistencia a la corrosión ${ }^{[4]}$. El proceso de proyección térmica $\mathrm{HVOF}$ es una de las mejores técnicas para la deposición de los polvos estándar de WC-Co y $\mathrm{CrC}-\mathrm{NiCr}$ ya que las elevadas velocidades y temperaturas relativamente bajas empleadas en este proceso, en comparación con la proyección por plasma, provocan una menor descomposición de los carburos durante el proceso de proyección, permitiendo la obtención de recubrimientos resistentes al desgaste, con la retención de un elevado porcentaje de material de refuerzo y muy baja porosidad ${ }^{[5}$ y 6 .

Por otra parte, el empleo de recubrimientos obtenidos por proyección térmica HVOF presenta ventajas, desde un punto de vista medioambiental, respecto a los recubrimientos electrolíticos empleados actualmente. El empleo de estos recubrimientos asegura la completa eliminación de los iones $\mathrm{Cr}^{+6}$ presentes en los baños galvánicos, lo que supone beneficios tanto para el medio ambiente como para la salud.

\section{MATERIALES Y PROCEDIMIENTO EXPERIMEN- TAL}

Los materiales estudiados en este trabajo han sido los recubrimientos de $\mathrm{CrC75}-(\mathrm{NiCr} 20) 25$ y WC86-Co10Cr4, depositados mediante proyección térmica HVOF, sobre un substrato de aluminio (EN AW-5754) con un espesor aproximado de 130 y $185 \mu \mathrm{m}$, respectivamente. La superficie del substrato fue pulida y chorreada con microesferas de vidrio (tamaño de las partículas entre 250 y 400 $\mu \mathrm{m})$ antes del proceso de deposición HVOF. Para proyectar el material se empleó una pistola C-CJS (Thermico, Alemania). En la tabla I se indican las características de los polvos y los parámetros de proyección.

Tabla I. Parámetros de proyección del proceso HVOF Table I. Spray parameters of HVOF process

\begin{tabular}{lcc}
\hline & CrC75NiCr25 & WC86-Co10Cr4 \\
\hline $\begin{array}{l}\text { Tamaño del polvo }[\mu \mathrm{m}] \\
\text { (aglomerado y sinterizado) }\end{array}$ & $-45 \div+15$ & $-30 \div+15$ \\
$\begin{array}{l}\text { Combustible (Queroseno) }[\mathrm{l} / \mathrm{h}] \\
\text { Gas portador }\end{array}$ & 8 & 11 \\
$\quad$ Nitrógeno $[\mathrm{l} / \mathrm{min}]$ & 25 & 25 \\
$\begin{array}{l}\text { Gases de combustión } \\
\text { Hidrogeno }\left[\mathrm{m}^{3} / \mathrm{h}\right]\end{array}$ & 3 & 3 \\
Oxigeno $\left[\mathrm{m}^{3} / \mathrm{h}\right]$ & 40 & 45 \\
\hline
\end{tabular}

La microestructura del recubrimiento se ha estudiado mediante microscopía electrónica de barrido. La determinación de la microdureza y de las propiedades elasto-plásticas de los recubrimientos se ha realizado mediante un ultra-microdurómetro Fischerscope H100, capaz de medir continuamente la fuerza aplicada y la profundidad de penetración, empleando indentador Vickers. Los ensayos se han realizado con valores de carga aplicada de 1.000 $\mathrm{mN}$, la cual se ha aplicado gradualmente, en 20 etapas, con un tiempo de reposo entre dos niveles de carga de 1 segundo. Los valores medios se han obtenido a partir de 25 indentaciones diferentes, descartándose los valores anómalos consecuencia de los defectos presentes en los recubrimientos.

La evaluación tribológica de los recubrimientos (coeficiente de fricción y velocidad de desgaste) se ha realizado empleando un tribómetro Pin-on-Disc acondicionado con una unidad de control de humedad y temperatura, de acuerdo con la norma de ensayos de desgaste ASTM G-99. Los ensayos se han efectuado con y sin lubricación (lubricante Repsol 15W40), a una velocidad lineal constante de $10 \mathrm{~cm} / \mathrm{s}$ aplicando valores de carga sobre el pin de $40 \mathrm{~N}$. Las condiciones ambientales se han ajustado a una temperatura de $25^{\circ} \mathrm{C}$ y una humedad relativa del $50 \%$. Con la finalidad de evitar el desgaste del pin, se ha empleado una bola de $6 \mathrm{~mm}$ de diámetro de WC-6\%Co. La velocidad de desgaste se ha calculado evaluando el material eliminado, mediante la medición del canal de desgaste con un rugosímetro-perfilómetro (Taylor-Hobson) ${ }^{[7]}$. Se han realizado un mínimo de tres ensayos para cada condición y material.

\section{RESULTADOS}

\subsection{Microestructura de los recubrimientos}

En la figura 1a) se muestra una micrografía SEM de una sección transversal del recubrimiento de CrC-NiCr obtenido por proyección térmica HVOF, con un espesor aproximado de $130 \mu \mathrm{m}$. En la figura 1b), se observa la microestructura del material constituida por una fase matriz (solución sólida $\mathrm{Ni}-20 \% \mathrm{Cr}$ ) junto con partículas de refuerzo (CrC).

El recubrimiento presenta la típica estructura laminar, con laminas deformadas de forma paralela al substrato de aluminio. Esta estructura es característica del proceso de proyección térmica HVOF, en el cual las partículas de polvo son aceleradas y calentadas a medida que se proyectan a través de la llama creada en la pistola HVOF. La parte externa de las 

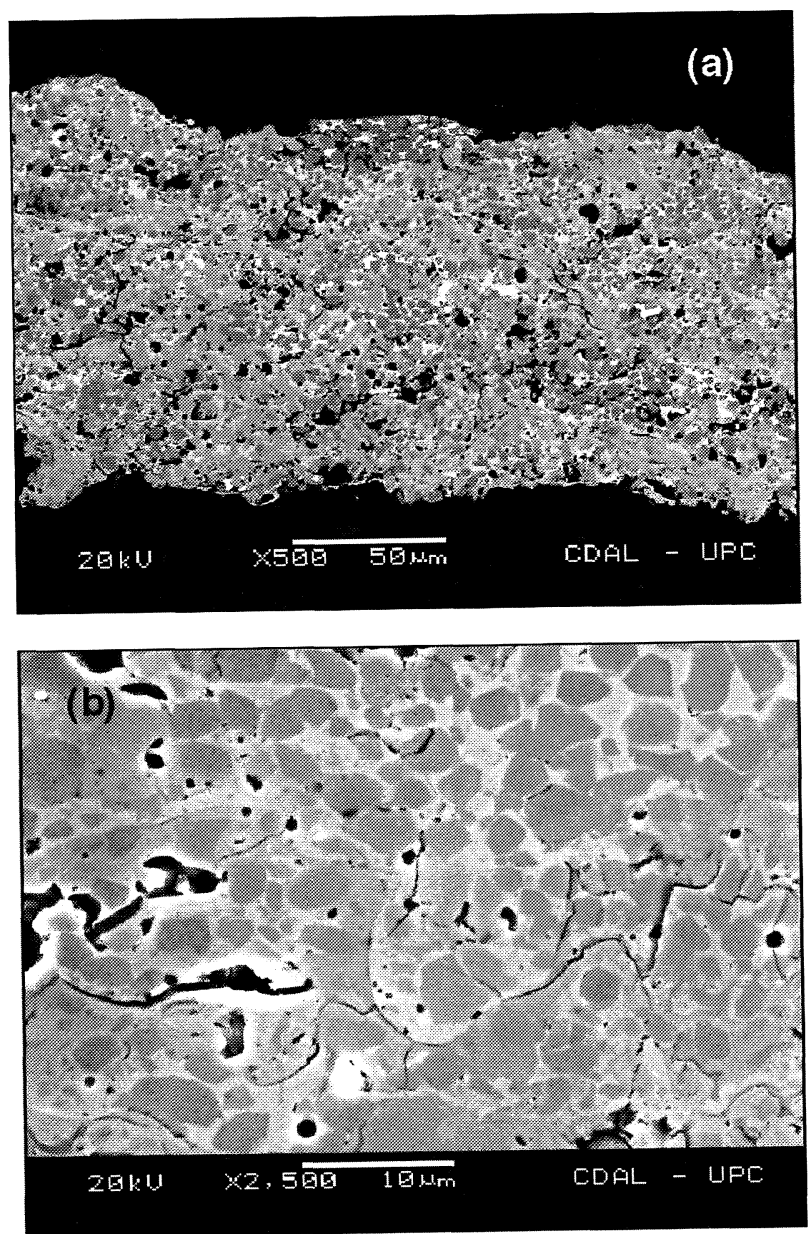

Figura 1. a) Micrografía SEM de la sección transversal de recubrimiento de $\mathrm{CrC75}(\mathrm{NiCr} 20) 25$ y b) detalle de la microestructura.

Figure 1. a) SEM micrograph of a cross-section of $\mathrm{CrC75}$ (NiCr20) 25 coating and b) microstructural detail.

partículas se funde, excepto las partículas de carburo, y el recubrimiento crece por el sucesivo apilamiento de las gotas que colapsan y se deforman en la superficie del material, experimentando al mismo tiempo un rápido enfriamiento. De acuerdo con la observación metalográfica (Fig. 2), el recubrimiento de $\mathrm{CCr}-\mathrm{NiCr}$ parece mostrar una buena adherencia sobre el substrato de la aleación de aluminio.

La figura 3a) se muestra una micrografía SEM de una sección transversal del recubrimiento de WC-CoCr obtenido por proyección térmica HVOF, con un espesor aproximado de $185 \mu \mathrm{m}$. En la figura $3 \mathrm{~b}$ ), se observa la microestructura del material constituida por una fase matriz (cobaltocromo) junto con partículas de refuerzo (WC).

El recubrimiento de WC-CoCr presenta una muy baja porosidad, mientras que el recubrimiento de $\mathrm{CrC}-\mathrm{NiCr}$ presenta una cierta porosidad, siendo necesario ajustar las condiciones de proyección

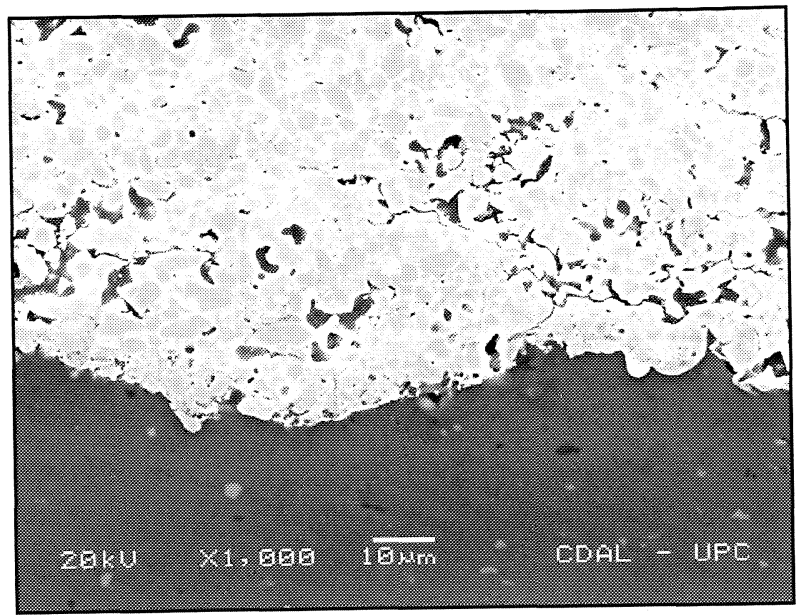

Figura 2. Interfase entre el substrato de aluminio y el recubrimiento de $\mathrm{CCr}-\mathrm{NiCr}$.

Figure 2. Interface between the $\mathrm{CCr}-\mathrm{NiCr}$ coating and the aluminium substrate.
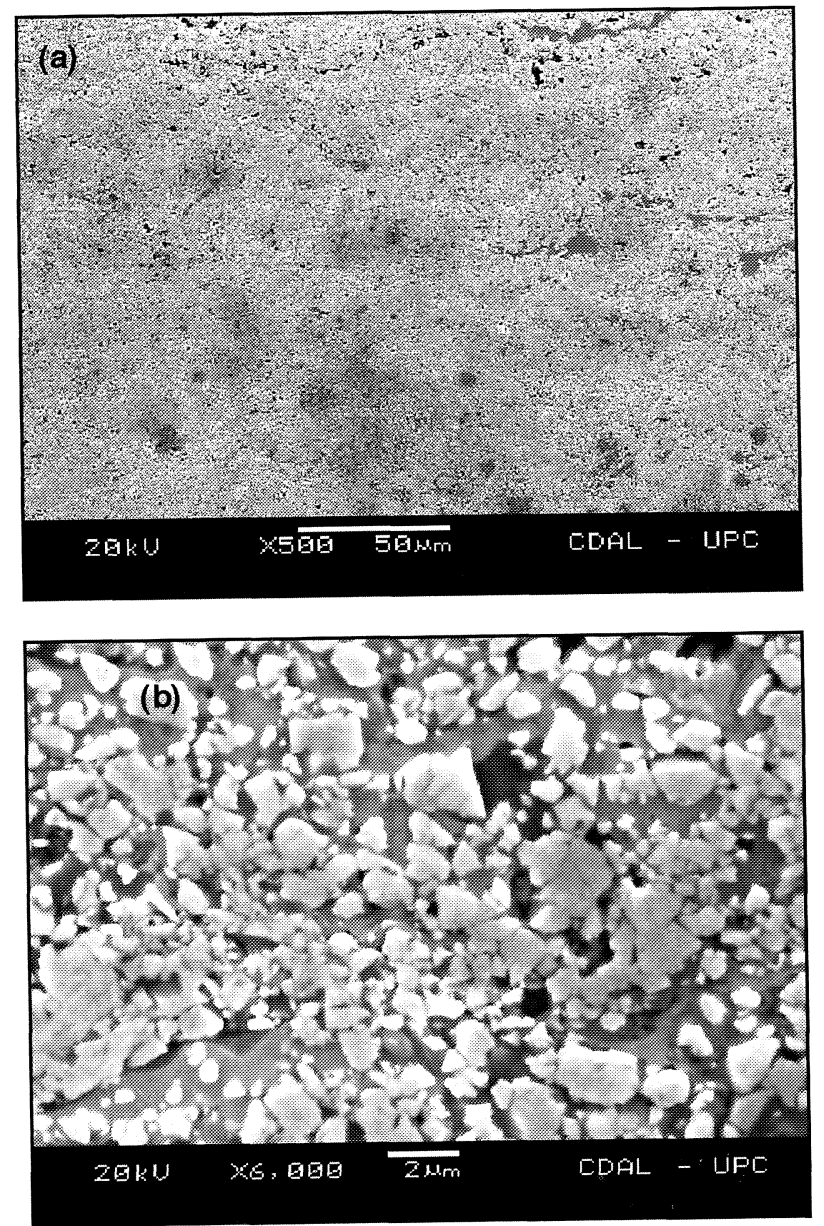

Figura 3. a) Micrografía SEM del recubrimiento de WC86Col0Cr4 y b) detalle de la microestructura.

Figure 3. a) SEM micrograph of WC86-Co10Cr4 coating and b) microstructural detail.

del recubrimiento de $\mathrm{CrC}-\mathrm{NiCr}$ para mejorar su calidad. 
Tabla II. Propiedades mecánicas y tribológicas (valores medios)

Table II. Mechanical and tribological properties (mean values)

\begin{tabular}{|c|c|c|}
\hline & $\mathrm{CrC75-NiCr25}$ & WC86-Co10Cr4 \\
\hline Dureza (HU / Hplas) [GPa] & $7,2 / 12,6$ & $8,7 / 14,8$ \\
\hline Módulo Elástico E/(1- $\left.v^{2}\right)[G P a]$ & 218 & 285 \\
\hline Recuperación elástica [\%] & 39,4 & 38,3 \\
\hline Rugosidad Ra $[\mu \mathrm{m}]$ & 4,9 & 4,5 \\
\hline \multicolumn{3}{|l|}{$\begin{array}{l}\text { Coeficiente de fricción } \\
\text { (valor medio) }\end{array}$} \\
\hline Ensayos con lubricación & 0,12 & 0,12 \\
\hline Ensayos sin lubricación & 0,21 & 0,20 \\
\hline \multicolumn{3}{|l|}{$\begin{array}{l}\text { Velocidad específica de } \\
\text { desgaste }\left[\mathrm{m}^{3} / \mathrm{m} \cdot \mathrm{N} \times 10^{-15}\right]\end{array}$} \\
\hline Ensayos con lubricación & 9,0 & 7,8 \\
\hline Ensayos sin lubricación & 28,2 & 12,3 \\
\hline
\end{tabular}

\subsection{Propiedades mecánicas y tribológicas}

Los ensayos de micro-dureza proporcionan valores de la carga aplicada y la profundidad de la penetración para los ciclos de carga y descarga. Los valores de microdureza calculados mediante este método ${ }^{[8]}$ corresponden a los valores de dureza bajo carga (Dureza Universal, HU) y dureza después de retirar la carga aplicada (Dureza plástica, Hplas). Este método, también permite determinar la recuperación elástica experimentada en el proceso de descarga así como estimar aproximadamente el módulo de cizalladura. La tabla II muestra los valores medios de los ensayos realizados en una sección transversal de los recubrimientos. En la tabla II también se indican los resultados de los ensayos tribológicos realizados así como la rugosidad superficial (Ra).

En la figura 4a) se muestra el canal de desgaste formado en la superficie del recubrimiento de WC-CoCr (ensayo Pin on disc sin lubricación) y en la Fig. 4b se puede observar un detalle del interior del canal de desgaste en la muestra de $\mathrm{CrC}$ $\mathrm{NiCr}$, en el cual se puede apreciar claramente la microestructura del recubrimiento.

Los recubrimientos de WC-CoCr son significativamente más duros que los de $\mathrm{CrC}-\mathrm{NiCr}$. Esta mayor dureza comporta un mejor comportamiento tribológico, mostrando un menor desgaste en comparación con el recubrimiento de CrC-NiCr. Así mismo, los recubrimientos de WC-CoCr presentan una menor rugosidad superficial como consecuencia del menor tamaño del polvo depositado.
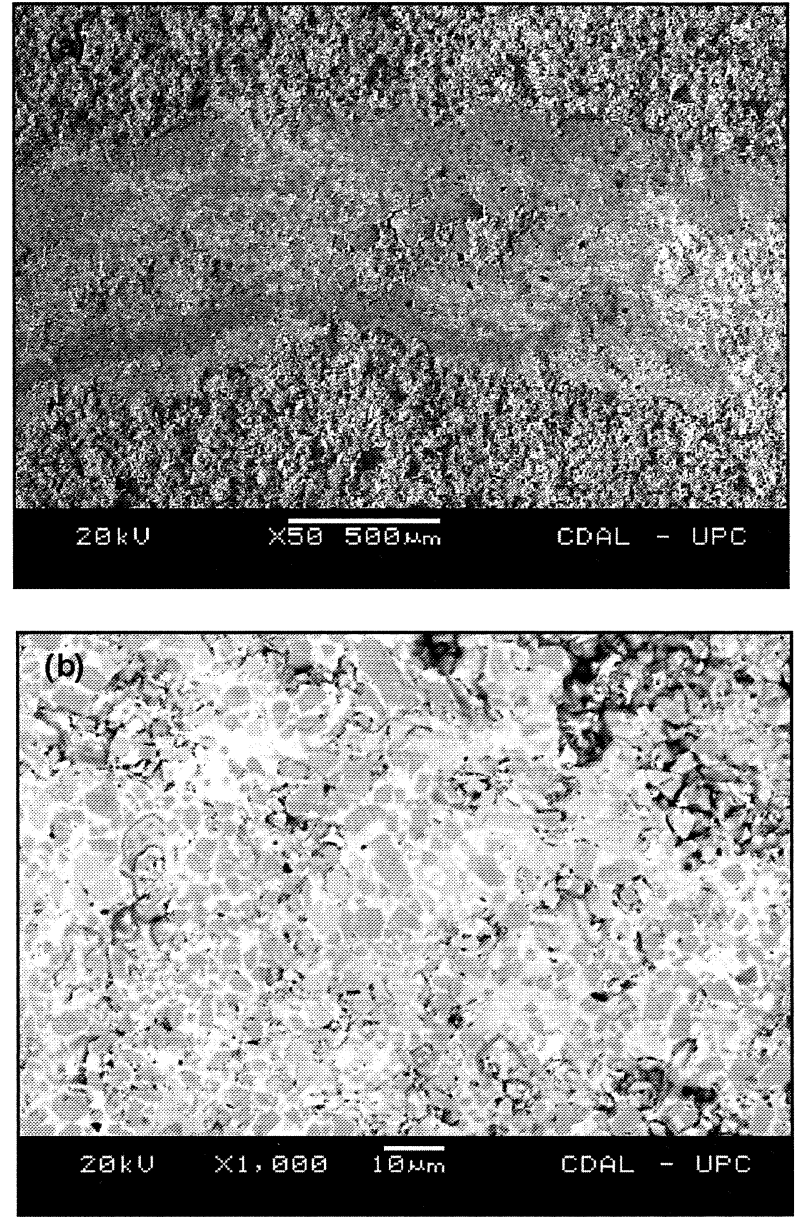

Figura 4. a) Micrografía SEM del canal de desgaste en el recubrimiento de WC-CoCr y b) detalle del interior del canal de desgaste en el recubrimiento de $\mathrm{CrC}-\mathrm{NiCr}$.

Figure 4. a) SEM micrograph of the wear track of the WC$\mathrm{CoCr}$ coating and b) wear track detail of the $\mathrm{CrC}-\mathrm{NiCr}$ coating.

Durante los ensayos Pin on disc pueden observarse oscilaciones en la evolución del coeficiente de fricción, mas acusadas en los ensayos en seco, las cuales son consecuencia de la formación de micro-contactos de carácter adhesivo entre el pin y el recubrimiento, así como por la producción de partículas en el canal de desgaste ("tercer cuerpo") que comportan un mecanismo de desgaste de tipo abrasivo. En la figura 3b) pueden apreciarse las consecuencias del desprendimiento de algunas partículas de $\mathrm{CrC}$ durante el ensayo.

\section{CONCLUSIONES}

Los recubrimientos de WC86-Co10Cr4 depositados mediante proyección térmica HVOF sobre un substrato de aluminio (EN AW-5754) son significativamente más duros que los correspondientes a 


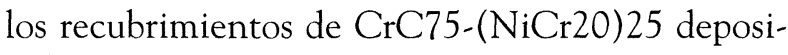
tados mediante el mismo proceso. Las mejores características mecánicas del recubrimiento de WC$\mathrm{CoCr}$, conjuntamente con una menor porosidad, comporta que estos recubrimientos presenten un mejor comportamiento tribológico (menor velocidad de desgaste) en comparación con el recubrimiento de CrC-NiCr. Esta diferencia es más acusada en los ensayos realizados sin lubricación. El mecanismo de desgaste, en ambos casos, es una combinación de un mecanismo de desgaste adhesivo conjuntamente con un mecanismo de desgaste abrasivo ocasionado por el desprendimiento de partículas de refuerzo. Los resultados obtenidos para estos recubrimientos son coherentes con los resultados presentados previamente para este tipo de recubrimientos depositados mediante el proceso HVOF sobre substratos de acero ${ }^{[9]}$.

La elevada velocidad alcanzada por las partículas proyectadas mediante el proceso de proyección térmica HVOF parece asegurar una buena adherencia de los recubrimientos estudiados sobre el substrato de aluminio (EN AW-5754). Este hecho, conjuntamente con las buenas características mecánicas y tribológicas obtenidas, permite proponer esta técnica y este tipo de recubrimientos como un método adecuado para mejorar las características superficiales de las aleaciones de aluminio que per- mita su empleo en aquellas aplicaciones donde estos componentes estén sometidos a elevadas solicitaciones de desgaste y fricción.

\section{REFERENCIAS}

[1] R. Schwetzke y H. KReye, J. Thermal Spray Technol. 8 (1999) 433-439.

[2] K.J. Stein, B.S. Schorr y A. Marder, Wear 224 (1999) 153-158.

[3] L. Russo y M. DoRfmann, Thermal Spraying: Current status and future trends. High Temperature Society of Japan, Osaka, Japan, 1995, pp. 681-686.

[4] Y. Fukuda y M. KumON, Thermal Spraying: Current status and future trends. High Temperature Society of Japan, Osaka, Japan, 1995, pp. 107-111.

[5] L. Ajdelsztajn, J.A. Picas, G.E. Kim, F. Bastian y J. Schoenung, Mater. Sci. Eng. A 338 (2002) 33-43.

[6] R. Schwetzke y H. KreYe, Proc. 15th Int. Thermal Spray Conference, Nice, France (1998) 187-192.

[7] B. BHuPHAN y B.K. GuPTA, Handbook of Tribology, Krieger Publishing Company, EE.UU., 1997, p. 1.168.

[8] J.L. Loubet, J.M. Georges y G. MeIlle, Microindentation Techniques in Materials Science and Engineering, ASTM STP 889 (1986) 72-89.

[9] D.A. Stewart, P.H. Shipway y D.G. Mccartney, Wear 225-229 (1999) 789-798. 